\title{
Can Heritage Bots Thrive? Toward Future Engagement in Cultural Heritage
}

\author{
Angeliki Tzouganatou
}

In the digital age, cultural organizations strive to retain audience engagement especially via experimentation with novel technologies and social media. The latter are increasingly influencing the way cultural heritage is perceived, providing options for grappling with crucial issues in the sector, including sustainability, openness, and public participation. One tool that has been deployed to explore these issues is the chatbot, a computer program designed to simulate conversation with human users, especially over the internet. Chatbots run through different conversational interfaces, but they have a particularly heavy application in Facebook Messenger. Within the museums and cultural sector specifically, these robotic media are regularly proclaimed to offer novel engagement mechanisms that can empower participants to actively participate in the heritage process. However, most heritage Messenger bots are purely informative and objector exhibit-centered, providing little opportunity for meaningful interactivity, creative expression, or critical engagement. This article explores and critically reviews three Messenger chatbots related to heritage organizations, concluding with suggestions for their future development.

Digital technologies and social media (Giaccardi 2012) are increasingly influencing and transforming the way cultural heritage is perceived. They provide solutions to crucial matters of concern for the archaeological and heritage sector, including its sustainability, its openness, and its public engagement. The purpose of this article is to capitalize on this potential by researching three Facebook Messenger chatbots related to cultural heritage and proposing possibilities for improvements that can enhance user engagement and offer opportunities for more interactive and meaningful conversation.

\section{BRIEF HISTORICAL BACKGROUND}

The chatbot is a computer program designed to simulate conversation with human users, especially over the internet. In the 1990s, Michael Mauldin (1994) invented the term ChatterBot, from the word chatter, meaning "superficial talk," and the word bot, an abbreviation for robot. In subsequent years, the term was then shortened to chatbot.
The history of intelligent machines such as chatbots can be dated at least as far back as the 1950s, when the British mathematician and pioneer in computer science Alan Turing (1950) contemplated the concept that machines can think. Turing's research focused on machines as intelligent devices with the capacity to think just as humans do (Hodges 2009), out of which he developed the groundbreaking Turing test. This "imitation game," as he called it, aimed to appraise a machine's ability to display intelligent behavior that would be equivalent to, or indiscernible from, human behavior (Hodges 2009; Turing 1950).

Joseph Weizenbaum was the first to create a conversational agent that could interact with a user via natural language: in 1964, he developed the computer program ELIZA (see Weizenbaum 1966), which emulated a psychotherapist (Figure 1). Weizenbaum programmed ELIZA to ironically mimic a Rogerian psychotherapist who "doesn't offer insights but rather keeps the person talking to examine their own thoughts, feelings, and beliefs" (Boutin 2017). Significantly, people became emotionally attached to the program and to the emotional replies that ELIZA offered them (Bayerque 2016; Weizenbaum 1976). As Weizenbaum stated, "ELIZA created the most remarkable illusion of having understood in the minds of the many people who conversed with it" (1976: 189). ELIZA is now considered the first chatbot in the history of computer science (even though it predates the coining of the term chatbot).

\section{CHATBOTS IN THE CULTURAL HERITAGE SECTOR}

Over time, the literature on conversational agents and intelligent virtual humans has grown very large (Gonzalez et al. 2017). However, as of yet, it is still quite limited in relation to the cultural heritage sector. This discrepancy is surprising, because in the digital era, chatbots are generally seen as advantageous tools that can facilitate communication, provide easier access to information, and integrate into the digital media/marketing strategy of institutions to help build and attract new audiences. Chatbots offer novel, immediate engagement mechanisms, and as texting becomes more and more popular, they are seen as 


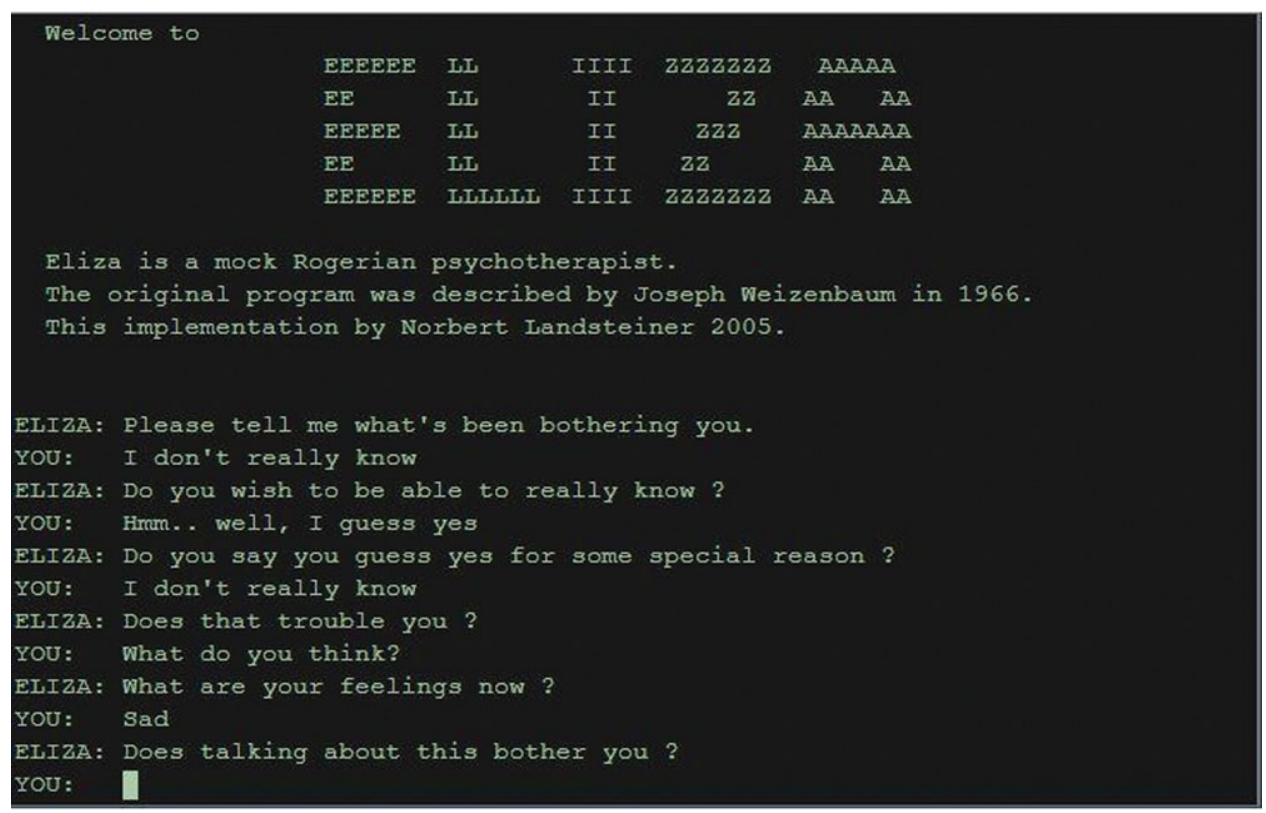

FIGURE 1. ELIZA's conversational flow, emulating a Rogerian psychotherapist (https://www.masswerk.at/elizabot/eliza.html).

useful in attracting people in multimodal ways. Moreover, they can operate both in a browser and on a mobile phone, and there is no need to install any additional apps.

On the other hand, there are also challenges that chatbots face. More particularly, chatbots are only available to people with internet access. Those that operate via messaging systems linked to specific social media platforms, such as Facebook Messenger, have an additional accessibility barrier: in order for users to be able to chat with the bot, they must have or create a profile on the social media platform. Furthermore, intellectually, it is not easy for chatbots to maintain meaningful conversations with users, as most of them have been created with the ability to provide small talk and not engage users in a deeper conversation. There are also technical problems with bots, such as the fact that they operate slightly differently in each web browser, and certain functions (e.g., ready-made replies) may cease to work properly depending on the user's browser. However, as chatbots are still in their early adoption phase, and their technology is rapidly evolving, these challenges may soon be overcome.

Although chatbots have tended to thrive within the customer service field, for example, sales (many stores such as American Eagle Outfitters and KAYAK use bots for helping their customers choose preferred goods), I wish to explore the possibilities for extending their offerings to cultural heritage institutions. As noted, chatbots are not a new concept, but after Facebook opened up its Messenger platform and application programming interfaces in 2016, the ground was paved for more developers to build chatbots, and thus Facebook Messenger bots inundated the Messenger platform.

Cultural heritage institutions were among those to take advantage of Messenger bots, although their application to date has arguably been quite limited. Many operate exclusively within the space of museums, with bots facilitating interaction between visitors and exhibits either by spoken natural language or via typed text. Bots typically serve as guides or as tools to help users plan their visit (e.g., by providing information regarding the museum's hours or by acting as museum docents). These chatbots could also be called "info bots," as their primary aim is to merely offer information to the visitor.

Below I review three key cultural heritage bots with the intent of exploring their strengths and weaknesses while suggesting future possibilities. Although chatbot work related to cultural institutions is still in its infancy, these three bots are pioneers and present novel features that make them stand out.

\section{ANNE FRANK HOUSE CHATBOT}

The chatbot of the Anne Frank House in Amsterdam, the Netherlands, which launched in March 2017, helps users plan their visit to the museum while also providing information about the life of Anne Frank and the events of World War II (Anne Frank House 2018). The bot is embedded in the Facebook page of the museum, and the chat experience is directed at off-site visitors, as there is no evidence that visitors would use it as an enhancement for their on-site experience.

The bot's content is curated by quick replies (Figure 2) that lead the user through predetermined conversational flows. The user can click through thematic options, and jump to the next topic, in an app-like manner (Shevat 2017).

It could be said that this predetermined "quick replies" option is a safe one, as by following the thematic options, the user interacts with the bot without asking any questions to which the bot may not be able to reply. Quick replies act as catalysts and follow-ups, working to circumvent disengagement and user 
Hi there, Angeliki! Thanks for visiting. I'm here to help you plan your visit to the Anne Frank House and learn more about Anne Frank's history.

Choose one of these options to get started:

Anne Frank has an impressive life story. Where would you like to start?

FIGURE 2. Anne Frank House chatbot's conversational flow, with ready-made replies at the bottom of the screen. Chatbot accessible with a Facebook account at m.me/annefrankhouse

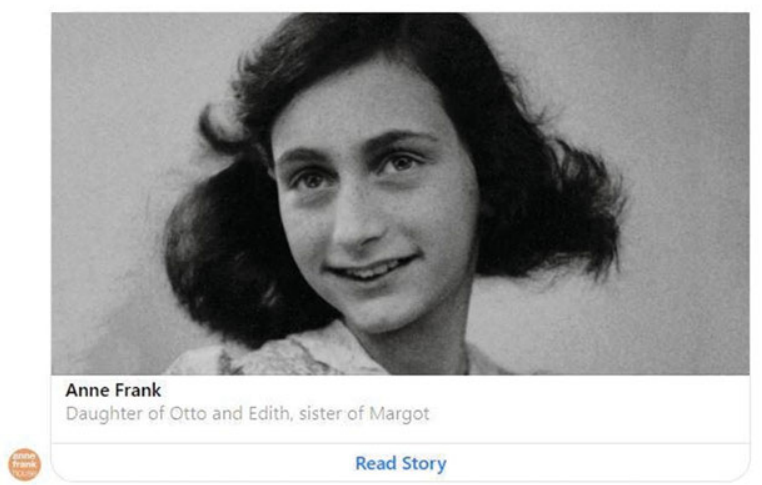

FIGURE 3. Anne Frank House bot is confused when a user asks about Anne Frank's father without referring to his full name, Otto Frank. Chatbot accessible with a Facebook account at m.me/annefrankhouse

frustration. While one might argue that it could be more frustrating and disengaging for users not to be able to ask their own questions through entering free text into the bot, the Anne Frank House has purposefully designed the bot to primarily use predefined answers and thus be "closed." In other words, the institution's aim with the bot is to ensure that its message is clear and that neo-Nazi terrorists or those with the potential to incite real violence are not able to spread their harmful voices (Baltussen 2017). However, the lack of free-form questions posed by the user, together with the fully guided conversational structure of the bot, urges the user to consume ready-made knowledge without really interacting on a personal level. Where users are able to type free-form questions, the bot can get confused (Figure 3), increasing the possibility for users to become disengaged and abandon their conversations.

\section{CASA IN CASA ADVENTOUR CHATBOT: THE HOUSE MUSEUMS OF MILAN}

The House Museums of Milan, Italy, is a group of four historical homes that launched a chatbot called "Di Casa in casa adventour," operating on a separate Facebook page from that of the main institution (Di Casa in casa adventour 2018). This chatbot is 
*Ambra Alfieri*

"Appunti di una vita senza fine" di Ambrogio Varesi da Rosate

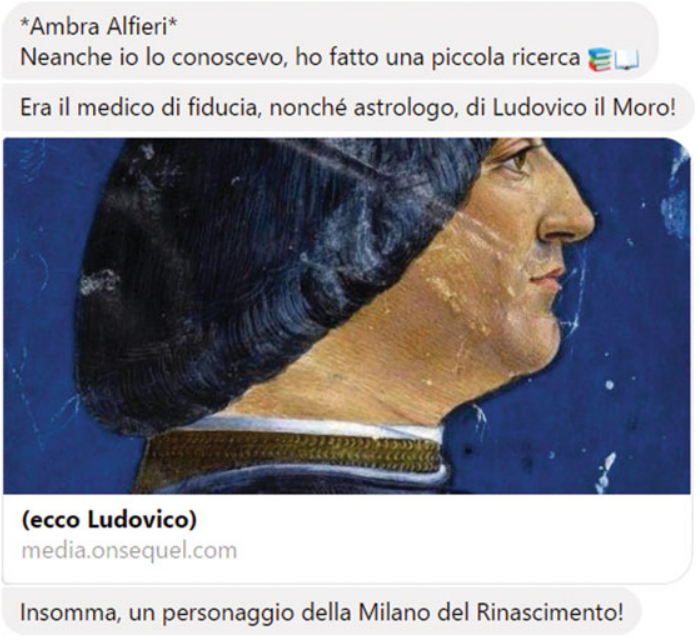

Dimmi di lui Posso aiutarti?

FIGURE 4. Di Casa in casa adventour chatbot's conversational flow, led by ready-made quick-reply buttons at the bottom of the screen. Chatbot accessible with a Facebook account at m.me/dicasaincasagame

one of a kind, proposing an alternative on-site learning experience through gamification. The user has the chance to explore the houses of the museum via an itinerary that requires finding hidden clues while fighting a Renaissance magician who was a real historical person (namely, the magician and doctor Ambrogio Varese da Rosate, who was the personal astronomer to "Ludovico il Moro," duke of Milan, in the fifteenth century; Figure 4; Giuliano Gaia, personal communication on August 30, 2018). The focus is on exploration of the museum rather than actual conversation with the bot, and thus the user is not detached from the physical place by the chatting experience. Instead the two spaces (the digital and the physical) are merged into one, delivering an interactive participatory experience. Information is presented in a seamless way, where the chatbot uses buttons exclusively (Figure 4), as well as different emojis and images from the museum's collection to help users find hidden clues. This approach helps motivate them and arouse interest across the experience.

The museum's aim with the bot is to attract new audiences, especially local young people (Boiano 2017; Boiano et al. 2018).

Among other things, evaluation results suggest that the game is highly entertaining and that participants found it useful as a learning tool, especially as a group experience rather than using it on their own (Boiano et al. 2018). Hence, a takeaway lesson from these findings might be that most info bots are unlikely to be very effective, because they provide little to no real participation opportunities: they typically do not encourage user/visitor collaboration, which has proved a significant element in the success of the Di Casa in casa adventour bot.

\section{MAXXI'S CHATBOT: THE NATIONAL MUSEUM OF THE 21ST CENTURY ARTS}

The chatbot of the National Museum of the 21st Century Arts (Museo nazionale delle arti del XXI secolo [MAXXI] 2018) in Rome, Italy (embedded in the museum's Facebook page), offers its users an alternative way to plan their visit and to explore both the collections and the incredible building where the museum is housed, designed by the pioneer architect Zaha Hadid. As a bilingual bot, which chats in Italian and in English, it offers a unique opportunity for both locals and tourists to engage. It is well curated, with the chatting experience led exclusively by ready-made quick replies, where users can learn more about their preferred topics by clicking on them. Also, it proposes art and architecture itineraries in the manner of a guide (Figure 5): users pick their preferred curated itinerary and follow a designated route through the museum, and the bot acts as an exhibition docent, providing information about selected artwork (Figure 6). The experience is augmented with the use of images, quizzes, GIFs, emojis, videos of the installation of artwork, and Museum Coins.

"Museum Coins" refer to MAXXI's virtual currency (Figure 7), which users can earn by solving quizzes or even watching videos. These coins can be spent at MAXXI, allowing users to buy goods at lower prices. These rewards seem an especially meaningful addition to the bot, offering motivation to users to engage 


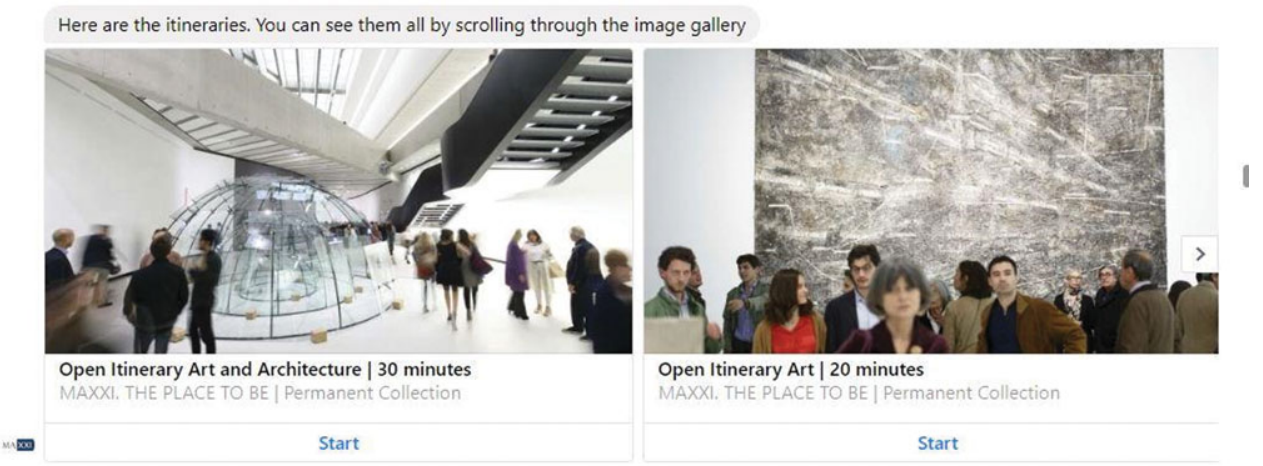

First of all, a piece of advice: pay attention to how works are displayed in the rooms

You will find paintings and photographs hung on the walls, but also floor installations - sometimes very large ones

Try and find the right distance between you and the works, to enjoy them without

threatening their safety

FIGURE 5. Art and architecture itineraries from the National Museum of the 21st Century Arts chatbot. Chatbot accessible with a Facebook account at m.me/museomaxxi

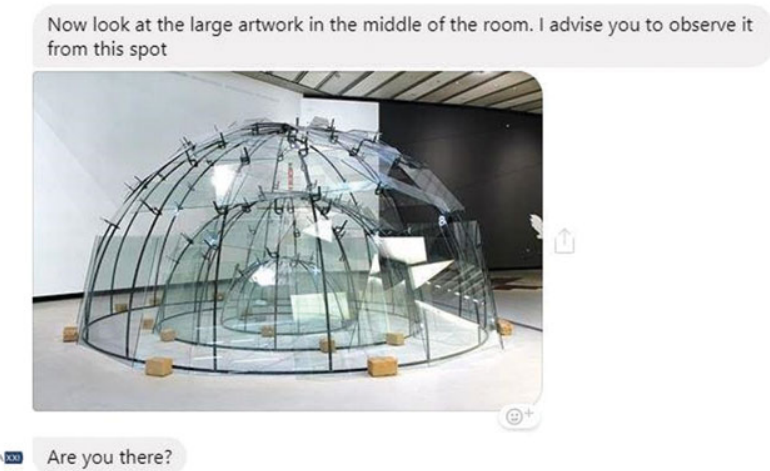

It is entitled "Untitled (Triple igloo)", and it is by Mario Merz, one of the major representatives of Arte Povera

Designed in 1984, it was then built by the artist in 2002; he passed away the following year

Want to know more?

FIGURE 6. Itinerary from the National Museum of the 21st Century Arts chatbot. Chatbot accessible with a Facebook account at m.me/museomaxxi

with the exhibits and perhaps even fostering repeat visits to the museum.

However, the chatting experience in MAXXI lacks meaningful interaction and input from users, as they can only consume readymade knowledge. The bot has no capacity to manage free-text inputs from users, so users could easily become disengaged (Figure 8). Moreover, as the conversation is exclusively guided, all control is held by the institution.

\section{WHAT'S NEXT? FUTURE POSSIBILITIES}

Of the bots discussed above, those with well-curated content and gamification elements appear the most engaging. But what can be done to further push the boundaries of heritage bots?

Indisputably, there is still much room for improvement. Such improvement might include the development of more 


\section{DIGITALREVIEW}

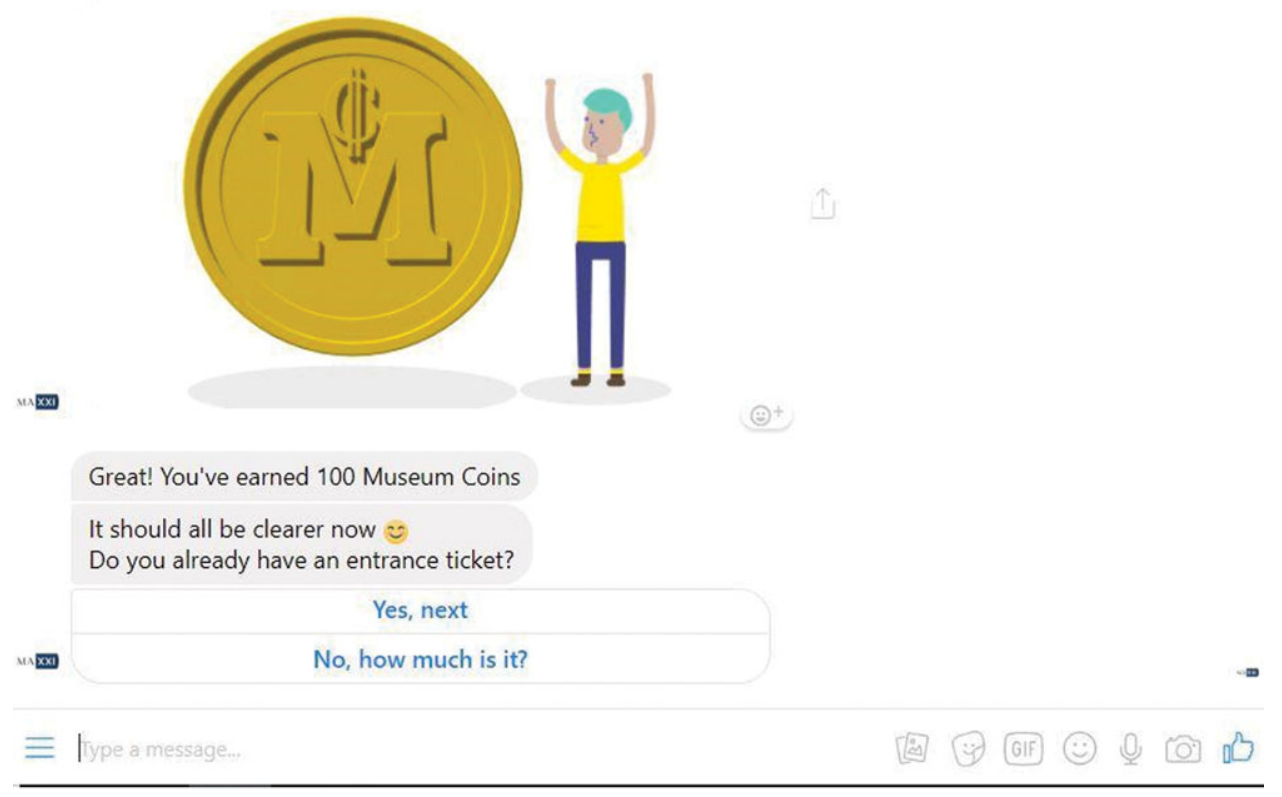

FIGURE 7. National Museum of the 21 st Century Arts virtual currency. Chatbot accessible with a Facebook account at m.me/museomaxxi

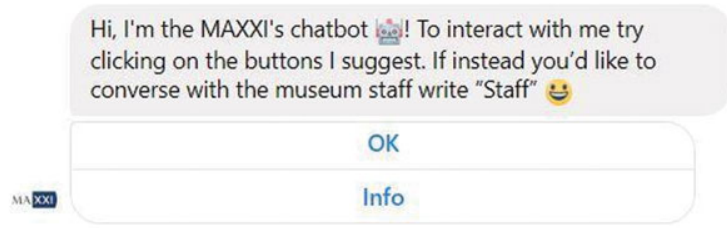

Sorry, I didn't understand your message. I'm just a bot to interact with me try clicking on the buttons I suggest. If instead you'd like to converse with the museum staff write m "Staff"

FIGURE 8. National Museum of the 21 st Century Arts bot is confused after a user types an answer rather than clicking on a ready-made reply. Chatbot accessible with a Facebook account at m.me/museomaxxi

sophisticated bots to encourage and foster dialogue between users, that is, proactive bots that can establish a bidirectional relationship between heritage sites and their audiences (Simon 2010). To do this, heritage institutions and bot developers would need to collaborate closely on the needs of the specific institution and its audience.

More do-it-yourself options might be inspired by Shawn Graham's (2017) call for digital media that are able "to move us, to inspire us, to challenge us," such as "bots of conviction," also known as "protest bots" (Sample 2014). Here certain thematic imperatives (e.g., topicality, uncanniness, oppositionality) are called upon to shape the development of bots that purposefully seek to provoke users about their values, beliefs, and prejudices. Graham provides tools and links to other resources that might allow us to create our own such protest bots on Twitter.

Ultimately, heritage bots have the potential to thrive if they push beyond merely delivering information. The majority of those in 
development and currently available online right now are closed and didactic in nature. The next step is to craft them into genuine mechanisms of chat, inviting users to actively interact and participate in the conversational experience.

\section{REFERENCES CITED}

Anne Frank House

2018 Chatbot. Electronic document,

https://www.facebook.com/annefrankhouse/, accessed July 2.

Baltussen, Lotte Belice

2017 Getting the Message Out There: Telling Anne Frank's Life Story with a Messenger Bot. Electronic document, https://www.slideshare.net/lottebelice/

museum-computer-group-2017-anne-frank-house-messenger-bot, accessed September 4, 2018.

Bayerque, Nicolas

2016 A Short History of Chatbots and Artificial Intelligence. Electronic document, https://venturebeat.com/2016/08/15/

a-short-history-of-chatbots-and-artificial-intelligence/, accessed July 12 , 2018

Boiano, Stefania

2017 Chatbots for Culture, Tourism and Exhibitions. Paper presented at Impact Hub, King's Cross (hosted by Deeson), London, May 24.

Boiano, Stefania, Ann Borda, Pietro Cuomo, Giuliano Gaia, and Stefania Rossi 2018 Chatbots in Museums: Hype or Opportunity? Museums and the Web 2018. Electronic document, https://www.museumsandtheweb.com/ chatbots-in-museums-hype-or-opportunity-\%E2\%80\%A8/, accessed July 5.

Boutin, Paul

2017 What Marketers Can Learn from a 1964 Bot. Electronic document, https://chatbotsmagazine.com/what-marketers-can-learnfrom-a1964-bot-6975871df45d, accessed August 11.

Di Casa in casa adventour

2018 Chatbot. Electronic document,

https://www.facebook.com/dicasaincasagame, accessed July 2.

Giaccardi, Elisa

2012 Heritage and Social Media: Understanding Heritage in a Participatory Culture. Routledge, London.

Gonzalez, Avelino J., James R. Hollister, Ronald F. DeMara, Jason Leigh, Brandan Lanman, Sang-Yoon Lee, Shane Parker, Christopher Walls, Jeanne Parker, Josiah Wong, Clayton Barham, and Bryan Wilder
2017 Al in Informal Science Education: Bringing Turing Back to Life to Perform the Turing Test. International Journal of Artificial Intelligence in Education 27(2):353-384.

Graham, Shawn

2017 An Introduction to Twitterbots with Tracery. Electronic document, https://programminghistorian.org/en/lessons/intro-to-twitterbots, accessed September 4, 2018

Hodges, Andrew

2009 Alan Turing and the Turing Test: In Parsing the Turing Test: Philosophical and Methodological Issues in the Quest for the Thinking Computer, edited by Robert Epstein, Gary Roberts, and Grace Beber, pp. 13-22. Springer, Dordrecht, the Netherlands.

Mauldin, Michael Loren

1994 ChatterBots, TinyMuds, and the Turing Test Entering the Loebner Prize Competition. In Proceedings of the Twelfth National Conference on Artificial Intelligence. Electronic document, https://pdfs.semanticscholar. org/bdd4/9b4a0b7de03b00412e3b807a855504e1d3af.pdf.

Museo nazionale delle arti del XXI secolo

2018 Chatbot. Electronic document,

https://www.facebook.com/museomaxxi/, accessed July 2, 2018.

Sample, Mark

2014 A Protest Bot Is a Bot So Specific You Can't Mistake It for Bullshit: A Call for Bots of Conviction. Electronic document, https://medium.com/ @samplereality/a-protest-bot-is-a-bot-so-specific-you-cant-mistakeit-for-bullshit-90fe10b7fbaa, accessed September 4, 2018.

Shevat, Amir

2017 Designing Bots. Creating Conversational Experiences. O’Reilly Media, Sebastopol, California.

Simon, Nina

2010 The Participatory Museum. Museum 2.0, Santa Cruz, California.

Turing, A. M.

1950 Computing Machinery and Intelligence. Mind 49:433-460. Electronic document, https://www.csee.umbc.edu/courses/471/papers/turing.pdf, accessed July 10, 2018.

Weizenbaum, Joseph

1966 ELIZA-A Computer Program for the Study of Natural Language Communication between Man and Machine. Communications of the ACM 9(1):36-45.

1976 Computer Power and Human Reason. W. H. Freeman, San Francisco, California.

\section{AUTHOR INFORMATION}

Angeliki Tzouganatou $\square$ PhD researcher, School of Humanities, University of Hamburg, Germany (atzouganatos@outlook.com) 